\title{
Successful Alliance of MTA and PRF for Treating Young Permanent Tooth: A Case Report
}

\author{
Meeti Charan, Hind Pal Bhatia, Shveta Sood, Naresh Sharma and Akshara Singh* \\ Department of Pedodontics and Preventive Dentistry, Manav Rachna Dental College, India
}

*Corresponding author: Akshara Singh, Senior Lecturer, Department of Pedodontics and Preventive Dentistry, Manav Rachna Dental College, Sectort 43, Delhi Surajkund Road, Faridabad, Haryana, Pin- 121004, India

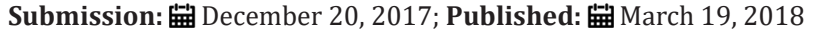

\begin{abstract}
This case report describes the treatment of an immature permanent tooth (open apex) with periapical lesion which was treated with MTA apexification and platelet rich fibrin (PRF) as an internal matrix. The root canal of immature permanent tooth was prepared and was disinfected with $2.5 \%$ Saline, $\mathrm{NaOCl}$ and EDTA. Triple antibiotic paste comprising Ciprofloxacin, Metronidazole and Tetracycline was given for intra radicular disinfection. When the tooth became asymptomatic, PRF was prepared and placed in the apical end of tooth followed by Mineral Trioxide Aggregate (MTA) as a sealing agent. It was obturated with thermoplasticized gutta percha and restored with Polycarbonate crown after 15 days. Progressive healing and reappearance of normal trabaculae pattern in periapical area was seen in 3 months follow up. Our findings suggest that PRF can be used for accelerating the healing process and for providing a barrier for the sealing agent like MTA in immature permanent teeth with periapical lesion.
\end{abstract}

Keywords: Immature permanent teeth; Mineral trioxide aggregate; Platelet rich fibrin; Triple antibiotic paste

\section{Introduction}

Young permanent teeth with pulpal necrosis require an artificial material to induce the closure of an open apex as the root development ceases. The closure of apex can be achieved by apexification which involves the formation of an apical barrier at the root apex in order to prevent the leakage of any microorganisms or toxic material to the surrounding periapical area, thus providing a hermetic seal [1]. Apexification with Mineral Trioxide Aggregate (MTA) offers major advantage over traditional Calcium hydroxide methods [2]. MTA is the preferred material for apexification as it reduces the treatment time, doesn't change the mechanical properties of dentine, stimulates repair, restores the tooth with a minimal delay and is highly biocompatible [3]. Despite the above mentioned advantages, overfill or under fill of MTA as an apical barrier may be disadvantageous. Lemon [4] introduced the "internal matrix concept" which involved the placement of hydroxyl apatite through the perforation to form an external barrier and matrix, against which the perforation repair material (amalgam) was condensed. He recommended the use of a matrix when the diameter of the perforation is larger than $1 \mathrm{~mm}$ to prevent the extrusion of sealing material. This concept was further modified by Bargholz [5] who recommended the use of collagen as a completely resorbable barrier material. The same concept is used for the placement of MTA as an apical barrier. Platelet rich fibrin (PRF) which is a second generation platelet concentrate can be used as a resorbable matrix material against which MTA apical barrier can be placed. PRF is an easily accessible source of growth factors to support bone and soft-tissue healing. It is added to surgical wounds which are in need of supported or accelerated healing. This case report describes the management of a necrosed young permanent tooth with open apex using MTA for apexification and PRF as an internal matrix.

\section{Case Presentation}

A 10 year old female patient complained of pain in upper front tooth region since one month. On taking detailed history it was revealed that the child had experienced dental trauma to anterior teeth six months back. Two days after the trauma they had availed dental services for the dental pain. However, the treatment was discontinued and follow up wasn't done by the guardians as the pain had subsided. After this anterior teeth remained asymptomatic until last month. History of present illness revealed that the nature of the pain was dull, constant, diffused with moderate severity which aggravated by intake of hot fluids. Intra-oral examination showed fracture involving the enamel in 11 and involving enamel, dentin and exposing pulp in 21. A large access opening was seen on the lingual side of 11 . On vertical percussion 21 was tender. Pulp vitality tests were done, which elicited negative responses in 21. Based on the above clinical signs and symptoms, a provisional diagnosis of complicated crown fracture leading to chronic apical periodontitis in 21 and uncomplicated crown fracture in 11 was proposed. Intra oral periapical radiograph revealed an incompletely formed apex with diffuse radiolucency (measuring $0.5 \times 0.5 \mathrm{~cm}$ ) in greatest dimensions with respect to 21 . It also showed radiopacity in $2 / 3 \mathrm{rd}$ of the canal of 21 which was indicative of intracanal medicament placed in the previous dental visit (Figure 1). Thus, a diagnosis of 
chronic periapical abscess in 21 was made. The treatment plan presented was to perform apexification in 21. Informed consent was taken by the guardians after explaining them the treatment planned in detail.

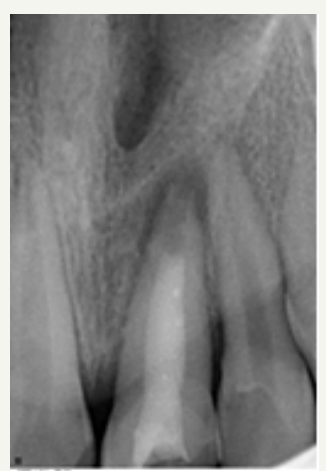

Figure 1: Periapical region of 21 showing open apex with radiolucency indicative of chronic apical periodontitis. The canal showed radiopacity in $2 / 3^{\text {rd }}$ of the canal, indicating intra-canal dressing in the canal.

\section{Treatment}

Local anaesthesia was administered after rubber dam application. Previously placed restoration and intra canal dressing was removed from the canal by saline irrigation. No.10 hand K file (Mani Inc., Tochigi, Japan) was used to check the patency of the canal. Biomechanical preparation was done using step back technique with stainless steel $\mathrm{H}$ files (Mani Inc., Tochigi, Japan) along with copious irrigation with saline, $2.5 \%$ sodium hypochlorite solution and EDTA (RC Help, Prime Dental, Thane, India) to ensure complete removal of the necrotic pulp tissue. Intracanal dressing of triple antibiotic paste consisting of Ciprofloxacin (250mg), Metronidazole (400mg) and Minocycline (100mg) in the ratio of 1:1:1 was placed according to the method followed by Prabhakar et al. [6] (Figure 2). After 40 days the tooth was asymptomatic but the periapical area showed no significant change in the periapical radiolucency. At this appointment, it was decided to enhance periapical healing by using PRF membrane as an internal matrix against which MTA would be placed as an apical barrier.

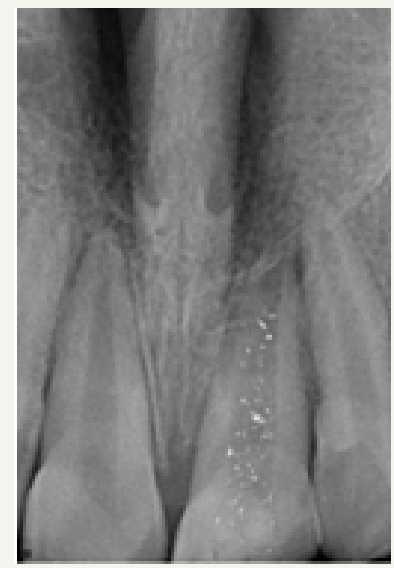

Figure 2: Triple antibiotic paste placed as an intra canal dressing in 21 .

\section{Preparation of PRF}

For the preparation of PRF membrane $10 \mathrm{ml}$ of venous blood was drawn from venipuncture of antecubital vein half an hour before the procedure. Blood was collected in a $10 \mathrm{ml}$ sterile glass tube without an anticoagulant and was immediately centrifuged at 3000 revolutions per minute for 10 minutes. After centrifugation three layers were seen the tube-topmost layer consisting of a cellular platelet poor plasma, PRF clot in the middle and red blood cells at the bottom (Figure 3a). The PRF clot was retrieved using tweezer and was placed on a sterile gauze piece to squeeze the excess fluid and obtain the PRF membrane (Figure 3b). An approximate size of the membrane was cut and placed in the canal using tweezer and hand pluggers. The matrix was placed at the apex, estimating its placement by the working length.
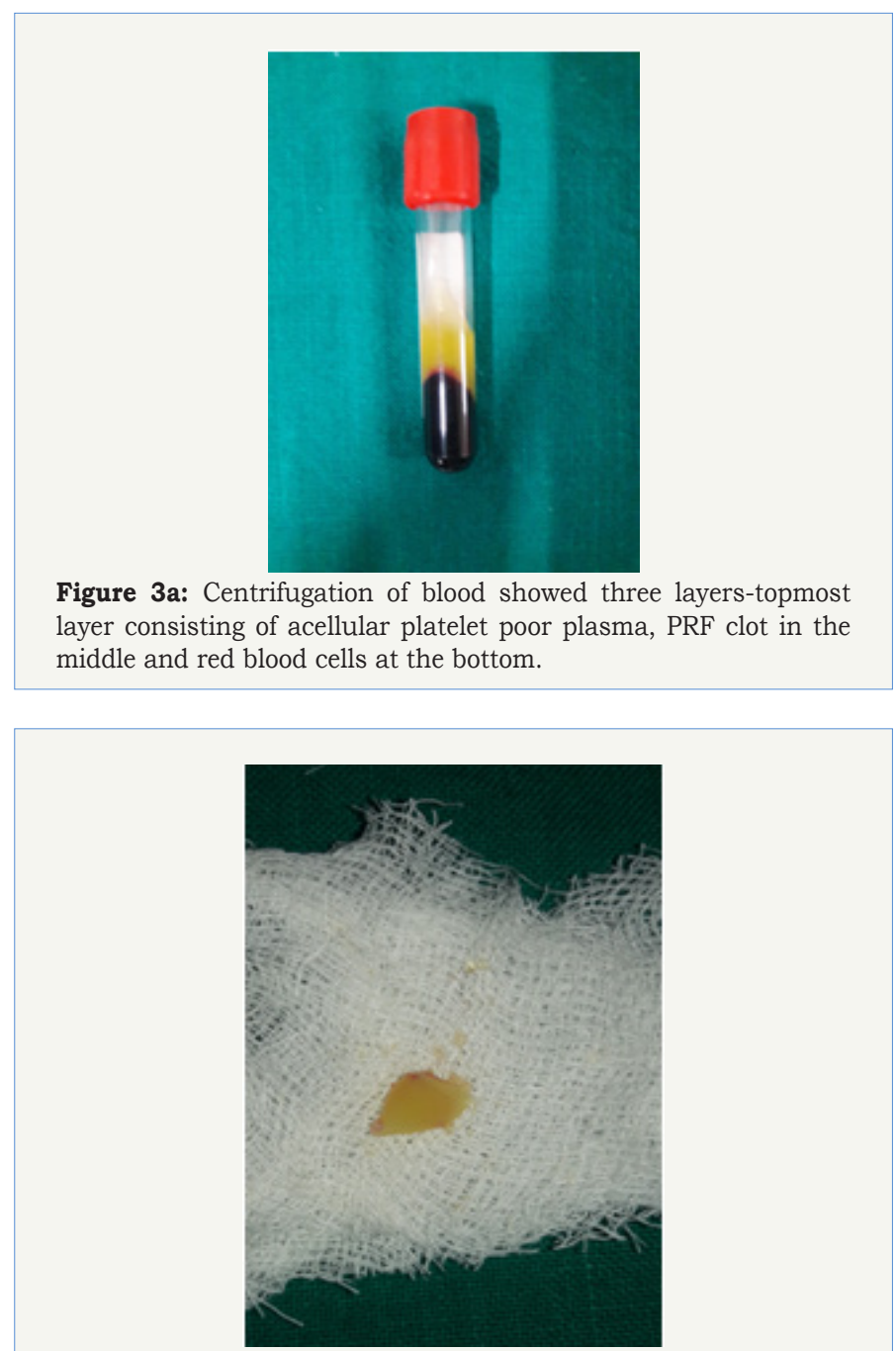

Figure 3b: The PRF clot after squeezing the excess fluid.

\section{MTA placement}

MTA (Angelus, Londrina, Brazil) was mixed as per the manufacturer's instructions and introduced into the canal and compacted using Schilder's pluggers (DENTSPLY Caulk, Milford, DE) against the PRF membrane. Digital radiograph was used to confirm adequate placement of MTA to form an apical stop approximately 
3-4mm thick. Moist cotton pellet was placed in the chamber and the access cavity was sealed with Intermediate Restorative Material (IRM) (Caulk/DENTSPLY, Milford, DE).

\section{Obturation and restoration}

After the patient remained asymptomatic for twenty four hours the tooth was isolated and accessed as before. A hand plugger was lightly tapped against the MTA plug to confirm a hardened set. The canal was obturated with thermoplasticized gutta perchaObtura III (Obtura Spartan Endodontics, Algonquin, USA) and AH Plus sealer (Dentsply DeTrey, Konztanz, Germany). The tooth was restored with resin composite (Figure 4). After one month recall the radiograph showed reappearance of normal trabaculae pattern in the periapical region of 21 . Considering the age of the child a temporary crown, Polycarbonate crown (3M, ESPE) was given in 21 (Figure 5).

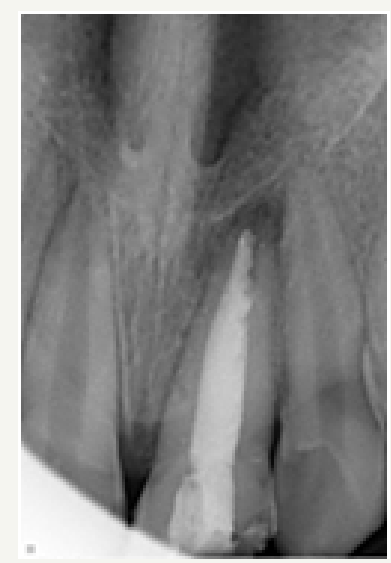

Figure 4: Internal matrix of PRF, $3-4 \mathrm{~mm}$ thick MTA and thermoplasticized gutta percha placed in 21 .

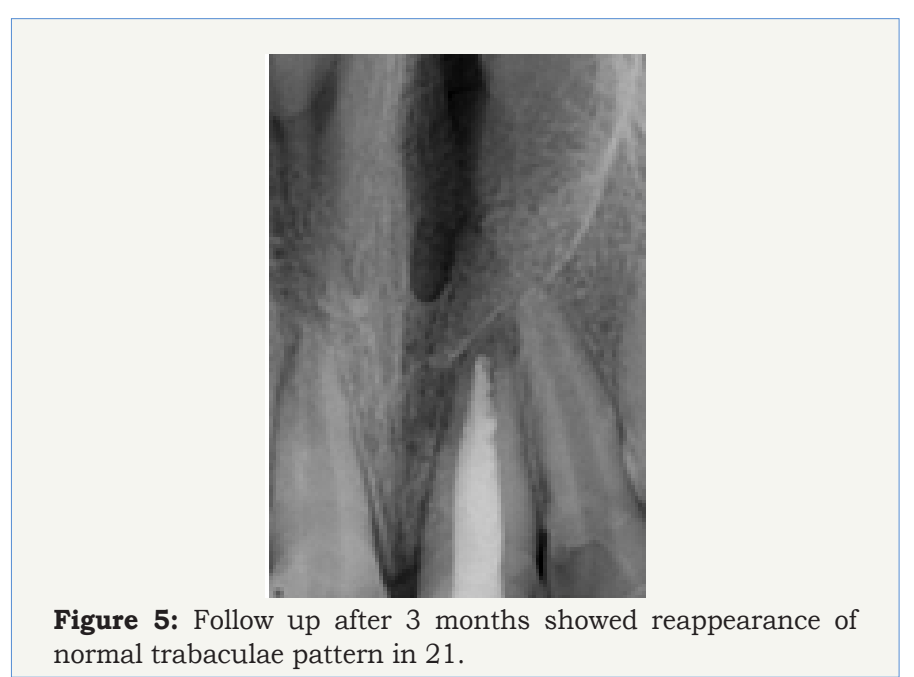

\section{Recall visits}

Progressive periapical healing and reappearance of normal trabaculae pattern in periapical area of 21 was confirmed through digital radiograph at 1, 2 and 3 months.

\section{Discussion}

The American Association of Endodontists defines apexification as a method to induce a calcified barrier in a root with an open apex or the continued apical development of an incomplete root in teeth with necrotic pulp [7]. Calcium hydroxide has been the material of choice for apexification and few studies have shown a $100 \%$ success rate with it but previous literature also states that Calcium Hydroxide can cause radicular dentin fracture when in contact with it for a long time [8]. Also the mean time required for the barrier formation is approximately 12-19 months [9]. Such long term treatment with multiple appointments reduces the compliance of the patients. Due to the above reasons the newer concept of single sitting apexification is now preferred. Triple antibiotic paste can be used as a dressing before the placement of MTA as the combination of Ciprofloxacin, Metronidazole and Minocycline is an effective for intra radicular disinfection. MTA has shown successful clinical and radiographic outcomes [10]. It also has shown good healing characteristics, like lack of inflammation, no ankylosis, cellular cementum formation (over growth), and PDL regeneration between the cementum and alveolar bone [11]. It takes an average of 3 to 4 hours for MTA to form a solid barrier thus allowing single sitting procedure. However; its complete setting may take up to 21 days [12]. The manipulation of MTA is difficult due to which its placement in the wide apical area is difficult to achieve [13]. A matrix can be used as a scaffold and a barrier for apexification procedures against which MTA can be placed and condensed. Calicum sulfate, hydroxyapatite, resorbable collagen, and platelet-rich fibrin are few materials that be used as a matrix for apexification [14]. PRF was chosen as an internal matrix because it is of an autogenous source and would have a favourable host tissue response. PRF produces leukocyte and platelets cytokine (PDGF, TGF and IGF-1) which are released as the matrix resorbs. These growth factors stimulate collagen production, induces anti-inflammatory reaction, recruits cells to the site of healing, increases vascular proliferation which in turn accelerates wound healing [15]. Thus in apexification procedure PRF can be used to promote wound healing and act as a barrier for the sealing material at the apical end. The combination of PRF and MTA is a revolutionary concept that should be preferred over the conventional apexification techniques for treating periapical lesions especially in children as it requires lesser clinical visits and accelerates wound healing. This also aids in developing a positive dental attitude in young children.

\section{References}

1. Maia Silveira CM, Nass Sebrão CC, Reis Vilanova LS, Sánchez-Ayala A (2015) Apexification of an immature permanent incisor with the use of calcium hydroxide: 16-year follow-up of a case. Case Rep Dent 2015: 984590 .

2. Huang GT (2009) Apexification: the beginning of its end. Int Endod J 42(10): 855-866.

3. Simon S, Rilliard F, Berdal A, Machtou P (2007) The use of mineral trioxide aggregate in one-visit apexification treatment: A prospective study. Int Endod J 40(3): 186-197. 
4. Lemon RR (1992) Nonsurgical repair of perforation defects. Internal matrix concept. Dent Clin North Am 36(2): 439-457.

5. Bargholz C (2005) Perforation repair with mineral trioxide aggregate: A modified matrix concept. Int Endod J 38(1): 59-69.

6. Prabhakar AR, Sridevi E, Raju OS, Satish V (2008) Endodontic treatment of primary teeth using combination of antibacterial drugs: an in vivo study. J Indian Soc Pedod Prev Dent 26(Suppl 1): S5-S10.

7. American Association of Endodontists (2003) Glossary of endodontic terms. $\left(7^{\text {th }}\right.$ edn $)$, American Association of Endodontists Chicago, USA.

8. Rafter M (2005) Apexification: A review. Dent Traumatol 21(1): 1-8.

9. Dominguez Reyes A, Muñoz Muñoz L, Aznar Martín T (2005) Study of calcium hydroxide apexification in 26 young permanent incisors. Dent Traumatol 21(3): 141-145.

10. Holden DT, Schwartz SA, Kirkpatrick TC, Schindler WG (2008) Clinical outcomes of artificial root-end barriers with mineral trioxide aggregate in teeth with immature apices. J Endod 34(7): 812-817.
11. Schwartz RS, Mauger M, Clement DJ, Walker WA (1999) Mineral trioxide aggregate: a new material for endodontics. J Am Dent Assoc 130(7): 967975.

12. Parirokh M, Torabinejad M (2010) Mineral trioxide aggregate: A comprehensive literature review-Part I: chemical, physical, and antibacterial properties. J Endod 36(1): 16-27.

13. Purra AR, Ahangar FA, Chadgal S, Farooq R (2016) Mineral trioxide aggregate apexification: A novel approach. J Conserv Dent 19(4): 377380

14. Goyal A, Nikhil V, Jha P (2016) Absorbable suture as an apical matrix in single visit apexification with mineral trioxide aggregate. Case Rep Dent 2016: 4505093 .

15. Simonpieri A, Del Corso M, Sammartino G, Dohan Ehrenfest DM (2009) The relevance of Choukroun's platelet-rich fibrin and metronidazole during complex maxillary rehabilitations using bone allograft. Part I: a new grafting protocol. Implant Dent 18(2): 102-111.
Creative Commons Attribution 4.0 International License

For possible submissions Click Here

\section{Submit Article}

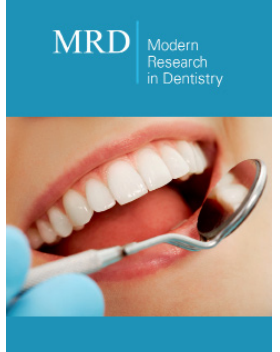

Modern Research in Dentistry

\section{Benefits of Publishing with us}

- High-level peer review and editorial services

- Freely accessible online immediately upon publication

- Authors retain the copyright to their work

- Licensing it under a Creative Commons license

- Visibility through different online platforms 